\title{
Genome-wide QTL mapping of nine body composition and bone mineral density traits in pigs
}

Sophie Rothammer ${ }^{1}$, Prisca V Kremer ${ }^{2,3}$, Maren Bernau ${ }^{3}$, Ignacio Fernandez-Figares ${ }^{4}$, Jennifer Pfister-Schär ${ }^{1}$, Ivica Medugorac ${ }^{1 *}$ and Armin M Scholz ${ }^{3}$

\begin{abstract}
Background: Since the pig is one of the most important livestock animals worldwide, mapping loci that are associated with economically important traits and/or traits that influence animal welfare is extremely relevant for efficient future pig breeding. Therefore, the purpose of this study was a genome-wide mapping of quantitative trait loci (QTL) associated with nine body composition and bone mineral traits: absolute (Fat, Lean) and percentage (FatPC, LeanPC) fat and lean mass, live weight (Weight), soft tissue X-ray attenuation coefficient (R), absolute (BMC) and percentage (BMCPC) bone mineral content and bone mineral density (BMD).
\end{abstract}

Methods: Data on the nine traits investigated were obtained by Dual-energy X-ray absorptiometry for 551 pigs that were between 160 and 200 days old. In addition, all pigs were genotyped using Illumina's PorcineSNP60 Genotyping BeadChip. Based on these data, a genome-wide combined linkage and linkage disequilibrium analysis was conducted. Thus, we used 44611 sliding windows that each consisted of 20 adjacent single nucleotide polymorphisms (SNPs). For the middle of each sliding window a variance component analysis was carried out using ASReml. The underlying mixed linear model included random QTL and polygenic effects, with fixed effects of sex, housing, season and age.

Results: Using a Bonferroni-corrected genome-wide significance threshold of $P<0.001$, significant peaks were identified for all traits except BMCPC. Overall, we identified 72 QTL on 16 chromosomes, of which 24 were significantly associated with one trait only and the remaining with more than one trait. For example, a QTL on chromosome 2 included the highest peak across the genome for four traits (Fat, FatPC, LeanPC and R). The nearby gene, ZNF608, is known to be associated with body mass index in humans and involved in starvation in Drosophila, which makes it an extremely good candidate gene for this QTL.

Conclusions: Our QTL mapping approach identified 72 QTL, some of which confirmed results of previous studies in pigs. However, we also detected significant associations that have not been published before and were able to identify a number of new and promising candidate genes, such as ZNF608.

\section{Background}

Domestication of the pig took place more than 9000 years ago. Since then, natural and artificial selection have led to the formation of many different breeds and strains. Currently, the pig is one of the most important livestock animals with more than 250 breeds worldwide. While most breeds (e.g. Large Black, Schwäbisch-Hällisch and Cerdo Iberico) serve mainly for commercial niche (high price)

\footnotetext{
* Correspondence: ivica.medjugorac@gen.vetmed.uni-muenchen.de ${ }^{1}$ Chair of Animal Genetics and Husbandry, Ludwig-Maximilians-University Munich, Veterinärstrasse 13, 80539 Munich, Germany

Full list of author information is available at the end of the article
}

markets, for large-scale commercial pork production, only a few breeds (e.g. Large White, Landrace, Pietrain, Duroc and Hampshire) are used [1,2]. These major breeds are of enormous economic interest and thus, it is not surprising that much effort has been put into Quantitative Trait Locus (QTL) mapping in pigs during the last two decades (reviewed by [1,3]). Today, there are almost 10000 QTL listed in the Pig QTL database (pigQTLdb, [4]; SS_10.2 downloaded on 04.02.2014). Among other reasons, insufficient resolution due to marker availability has limited traditional QTL analyses (many QTL cover more than $20 \mathrm{cM}$ ), which has delayed identification of causal genes or variants 
[5]. However, recently, the availability of cost-effective genome-wide SNP genotyping has made it possible to map QTL more accurately and this has increased opportunities to identify candidate genes and/or to implement genomic selection. During the last years, the first genome-wide association studies using Illumina's Porcine SNP60 Genotyping Beadchip were published (e.g. [6-10]). On the one hand, pig mapping data are important for long-term efficient pig breeding that integrates animal welfare. On the other hand, the pig is an extremely relevant animal model since it shares many genetic and physiological similarities with humans [11]. Thus, candidate regions/genes that are mapped for distinct traits in the pig can also be important for similar traits in humans and vice versa. In particular, loci associated with fatness and fat composition in pig can be relevant for the study of human obesity and obesityassociated diseases that are some of the most severe health issues $[6,8,12,13]$.

In this study, we carried out a genome-wide (44 809 SNPs) combined linkage and linkage-disequilibrium analysis (LDLA) in a dataset of 551 pigs that were a mixture of the following breeds in various proportions: Large Black, Pietrain, Duroc, Schwäbisch-Hällisch, Cerdo Iberico, European Wild boar, and Hampshire. The whole body traits that were analyzed included four traits for body composition (Fat g, FatPC \%, Lean g, LeanPC \%), three traits for bone mineral content and density (BMC g, $\mathrm{BMCPC} \%, \mathrm{BMD} \mathrm{g} / \mathrm{cm}^{2}$ ), the soft tissue X-ray attenuation coefficient (R) and live weight (Weight g). Except for Weight, all traits were obtained by Dual-energy X-ray absorptiometry (DXA), as described in Scholz and Förster [14] and in Kremer et al. [15]. Although candidate gene analyses for leg weakness and bone mineral traits have been performed at local levels (e.g. [16-18]), to our knowledge, this is the first genome-wide QTL mapping analysis for whole body bone mineral traits in pigs. Bone mineral traits are of special interest in pig production since they have been shown to be associated with osteochondrosis [17] and bone fracture risk [16]. Thus, these traits are part of the multifactorial leg weakness syndrome which is a serious animal welfare issue and moreover causes considerable economic losses [18-20]. Significant results (genome-wide $P$-value $<0.001$ ) of the genome-wide QTL mapping of these nine traits will be important for pig breeding. In addition, we identified QTL for Fat and FatPC that might represent regions of special interest for future analysis of obesity-related traits in humans.

\section{Methods}

\section{Ethical statement}

The trials reported in this work comply with the ethical guidelines of the Ludwig-Maximilians-University (LMU) of Munich and were conducted with the approval of the appropriate ethics committee of the District Government of Upper Bavaria, Germany (No. 55.2-1-54-2532.2-60-07 and No. 55.2-1-54-2531.2-22-08). Moreover, blood sampling was conducted only by certified veterinarians who follow the German Animal Welfare Act to avoid any unnecessary pain, suffering and damage to the animals.

\section{Animal samples}

For this study, 554 blood samples of individuals between 160 and 200 days old were collected at the Livestock Center of the Faculty of Veterinary Medicine (Oberschleissheim, Germany). The animals sampled represented six different breeds (Duroc, Cerdo Iberico, Large Black, Hampshire, Pietrain, Schwäbisch-Hällisch) and included both purebred and crossbred individuals. In addition to these six breeds, crossbred animals included varying proportions (0 to $25 \%$ ) of European Wild boar.

For all individuals, weight was measured with a mechanic livestock scale for weights between 1 and $250 \mathrm{~kg}$ and eight body composition traits were measured by dual energy X-ray absorptiometry (DXA) using a pencil beam scanner "GE Lunar DPX-IQ" with the whole body mode "adult normal” (Lunar software version 4.7e, GE Healthcare, Pittsburgh, PA, USA), as described in $[14,15]$. Values were predicted for bone mineral content in $\mathrm{g}(\mathrm{BMC})$ and percentage (BMCPC), bone mineral density in $\mathrm{g} / \mathrm{cm}^{2}$ (BMD), fat tissue mass in $\mathrm{g}$ (Fat) and percentage (FatPC), lean tissue mass in $\mathrm{g}$ (Lean) and percentage (LeanPC), and the X-ray attenuation coefficient (R). Prior to DXAscanning, animals were fasted for 16 hours. They were sedated by intramuscular injection of azaperone (Stresnil ${ }^{\odot}$ : $1.2 \mathrm{mg} / \mathrm{kg}$ body weight) followed by ketamine (Ursotamin ${ }^{\circ}$ : $40 \mathrm{mg} / \mathrm{kg}$ body weight) and then, an intravenous catheter was placed in an ear vein to continue ketamine administration if necessary.

\section{Genotyping data and quality control}

DNA extraction from blood samples was performed using the QIAamp DNA Blood Mini Kit from Qiagen. DNA of all sampled pigs was genotyped for 62163 single nucleotide polymorphisms (SNPs) using version 1 of the Porcine SNP60 Genotyping BeadChip (Illumina Inc., San Diego, USA). The physical positions of all SNPs were downloaded according to the reference assembly SGSC Sscrofa10.2/ susScr3 [21]. Markers that met one of the following criteria were excluded from further analysis: (i) successful genotyping results in less than $95 \%$ of the animals, (ii) frequent paternity conflicts in animals with known paternity, (iii) unknown or non-unique position according to the reference assembly susScr3, (iv) markers with a heterozygosity level less than 0.05 and (v) markers on porcine chromosomes $\mathrm{X}$ and $\mathrm{Y}$ since we concentrated on autosomes only. After this filtering process, the marker dataset comprised 44 809 markers. 
From the initial animal set, three individuals were excluded from further analysis because of a genome-wide genotyping call rate below $95 \%$ resulting in a final animal set of 551 animals.

\section{Reconstruction of haplotypes}

For haplotype reconstruction and imputation of missing genotypes, the Hidden Markov Model implemented in BEAGLE 3.0.4 was used [22]. Since additional animals and pedigree information improve the accuracy of haplotype reconstruction, we added another 49 animals that were otherwise not relevant for this analysis, and assigned all animals to three different groups: (i) 169 parent-offspring trios, (ii) 251 parent-offspring pairs and (iii) 151 unrelated individuals without any genotyped parent or offspring. It should be noted that some animals can be offspring in a pair and parent in a trio. Thus, the sum of all individuals in pairs, trios and the unrelated group exceeded the actual number of animals in the dataset.

\section{Unified additive relationships and principal component analysis}

To correct for population stratification and family relationships within the mixed linear model used for QTL mapping (see below), we estimated the unified additive relationships (UAR) between all animals [23]. Subsequently, all principal components of the UAR matrix were estimated using $\mathrm{R}$ [24]. The number of principal components to be integrated in the linear model was determined using the $R$ package paran [25], which is an implementation of the empirical method of Horn's parallel analysis. Based on the so-defined 18 principal components, we were able to efficiently reduce the initial matrix dimensions from $551 \times 551$ to $18 \times 551$, without significant loss of information since these 18 principal components explained more than $90 \%$ of the genetic variance.

\section{Locus IBD and diplotype relationship matrix}

Besides genome-wide relationships, local haplotype relationships were also included in the mixed linear model for QTL mapping. Genome-wide relationships were accounted for by using the 18 most significant principal components of the UAR matrix, while for local haplotype relationships we used sliding windows of 20 consecutive SNPs along the genome (larger windows of e.g. 40 SNPs also performed well and showed very similar results to the 20-SNP windows; data not shown). At each window-midpoint (i.e. between markers 10 and 11), the locus identity by descent (LocIBD) was estimated based on the method described in Meuwissen and Goddard [26]. The resulting haplotype-based IBD matrices were then converted into diplotype relationship matrices $\left(\mathbf{D}_{\mathbf{R M}}\right)$ using the procedure described for additive genetic relationship matrices at a QTL ( $\mathbf{G}_{\mathbf{R M}}$ matrix) in Lee and Van der Werf [27].

\section{Genome-wide QTL mapping}

QTL mapping was carried out by a procedure that is equivalent to the combined linkage/linkage disequilibrium mapping method reported in Meuwissen et al. [28]. While linkage information was accounted for during reconstruction of haplotypes based on available relationships, linkage disequilibrium is considered in the estimation of LocIBD. Finally, a variance component analysis in the middle of each of the 20-SNP sliding windows was performed in ASReml [29]. The mixed linear model included random QTL effects based on $\mathbf{D}_{\mathbf{R M}}$, as well as the fixed effects of sex, housing, season, and the covariates of age at sampling and the 18 principal components to account for polygenic effects. Effects of additional corrections, such as pedigree-based breed compositions, were also tested, but since they were not significant, they were omitted from the final mixed linear model. The resulting model was:

$$
\mathbf{y}=\mathbf{X} \boldsymbol{\beta}+\mathbf{Z q}+\mathbf{e}
$$

where $\mathbf{y}$ is a vector of phenotypes of the investigated trait, $\boldsymbol{\beta}$ a vector of fixed effects (including overall mean $\mu$, fixed effects, age at sampling and the 18 principal components), q a vector of random additive genetic effects due to QTL with $\mathbf{q} \sim \mathrm{N}\left(0, \mathbf{D}_{\mathbf{R M p}} \sigma_{q}^{2}\right)$, where $\mathbf{D}_{\mathbf{R M p}}$ is the diplotype relationship matrix at position $p$ of the putative QTL and $\mathbf{e}$ a vector of random residual effects with $\mathbf{e} \sim \mathrm{N}\left(0, \mathbf{I} \sigma_{e}^{2}\right)$, where I is an identity matrix.

The random effects $\mathbf{q}$ and $\mathbf{e}$ were assumed to be uncorrelated and normally distributed and their variances $\left(\sigma_{q}^{2}, \sigma_{e}^{2}\right)$ were simultaneously estimated using ASReml [29].

Using the logarithm of the likelihood estimated by ASReml for the model with $\left(\log L_{\mathrm{P}}\right)$ and without QTL effects ( $\log \mathrm{L}_{0}$; corresponding to the null hypothesis), we calculated the likelihood ratio test statistic $\left(\mathrm{LRT}=-2\left(\log \mathrm{L}_{0}-\log \mathrm{L}_{\mathrm{P}}\right)\right)$, which is known to be $X^{2}$-distributed with one degree of freedom [30]. In order to keep the false discovery rate low, we chose a conservative significance threshold of LRT $=31.275$, which corresponds to a $P$-value less than $2.24 * 10^{-8}$ before correction for multiple-testing and a Bonferroni-corrected genome-wide $P$-value less than 0.001 based on 44611 tested windows $\left(0.001 / 44611=2.24^{*} 10^{-8}\right)$. For each peak that exceeded this LRT threshold, we determined the confidence interval $(\mathrm{CI})$ of the position of the QTL using the 2-LOD ( $\log$ of odds; 1 LOD $=4.605$ LRT) drop-off criterion [5,31]. Overlapping CI of neighboring peaks for the same trait were combined into a single CI. In such cases, only the peak with the highest LRT was reported for the combined CI. Overlapping CI for different traits were assigned to a common QTL. For all genes within a QTL, a literature study was conducted to identify 
the QTL's candidate gene(s). As candidates we considered genes that fulfilled one of the following criteria: (i) genes within QTL for the same or related traits identified in previous studies in pig, (ii) known to be associated with related traits in other mammals or species and (iii) involved in pathways related to the investigated trait.

\section{Results}

\section{Genome-wide QTL mapping}

As shown in Figure 1, significant associations of distinct chromosomal regions with phenotype were found for each of the nine traits investigated, except for BMCPC. However, the number of identified QTL varied considerably from 2 for BMD to 41 for FatPC. Chromosomes 8 and 14 showed no significant associations while the remaining chromosomes had up to 31 (SSC6) significant associations. Table 1 shows a summary of the significant associations and their confidence intervals (CI) identified per trait and chromosome. A total of 195 associations were identified for all traits, which represented 72 QTL. The CI of only 24 of the 195 associations did not overlap with the CI for another trait (See Additional file 1).

Based on overlaps of CI between traits, two QTL stood out, i.e. one QTL on chromosome 6 between 7006410 and $14176920 \mathrm{bp}$ and one QTL on chromosome 2 between 129544104 and $135064176 \mathrm{bp}$. Within the region on chromosome 6 , the $\mathrm{CI}$ of seven traits (BMC, Fat, FatPC, Lean, LeanPC, R, Weight) overlapped and Weight had its highest LRT-value across the genome (53.279) in this region at position 8723052 . The region on chromosome 2 included five significant traits (Fat, FatPC, LeanPC, R, Weight). Each of these traits, except Weight, had its highest LRT-values across the genome (54.78-58.1) in this region and at exactly the same position (133 $237232 \mathrm{bp}$ ).

Peak-positions and $\mathrm{CI}$ of the highest genome-wide association for each trait are in Table 2. While BMC and BMD shared the highest peak at the same position (37 $403520 \mathrm{bp}$ ) in another region of chromosome 6, the greatest LRT for Lean (44.279) was on chromosome 12 (42 949544 bp). Table 2 also lists candidate genes for each QTL. These candidates and additional interesting candidate genes will be discussed in the next section.

\section{Discussion}

It has been shown that a meta-analysis that combines data from multiple populations improves both power and resolution of QTL mapping (reviewed by [3]). This raises the obvious question of whether combining multiple populations in a single-QTL mapping study will also provide more significant results. As for F2 populations in line-cross models [3], animal datasets that contain diverse populations and crosses should allow the mapping of QTL that are fixed in a population. In this paper, we show that diverse animal datasets such as the one used in this study can confirm many previously published QTL and, moreover, identify new unknown QTL.

Overall, we found that most of the identified QTL (48 out of 72) affected more than one trait, which is not surprising since the traits studied are not completely independent. While relationships of Fat or Lean with the percentage traits FatPC and LeanPC, as well as with Weight are obvious, there is also a linear relationship between FatPC and ratio of the mass attenuation coefficients of soft tissue ( $\mathrm{R}$ value $=\mathrm{X}$-ray attenuation coefficient) [32-34]. Moreover, it has been extensively shown in humans that obesity correlates with bone metabolism (reviewed by $[35,36])$. We will come back to these relations when discussing possible candidate genes (full names of all gene symbols mentioned hereafter are in Additional file 2).

It is worth mentioning that some of the mapped QTL lie in the vicinity of an even stronger QTL for the same trait (s). In such cases, further investigations, such as multipleQTL analyses, are needed to clarify if these are separate QTL or rather represent carryover effects of nearby large QTL $[37,38]$. Nevertheless, for several of these "possible carryover QTL", there is some indication that they are separate QTL since promising candidate genes could be defined within the CI of some QTL. For example on chromosome 7, there are seven non-overlapping CI for Fat and/or FatPC (referred to as CI1 to CI7 in the following) within a segment of $31 \mathrm{Mb}$ (between 99352936 and $130971280 \mathrm{bp})$. Although, some of these could be caused by carryover effects, we were able to identify candidate genes within each $\mathrm{CI}$, some of which were reported in previous QTL studies in pigs or were associated with functions related to body composition in pigs and other mammals: (i) SIPA1L1 and DPF3 are good candidates for CI1 (between 99352936 and 102221456 bp) since the chromosomal segment containing these genes was reported to be associated with $10^{\text {th }}$ rib backfat in pig [39]; (ii) for CI2 (between 104522416 and 105871296 bp), TGFB3 was identified as a candidate gene since it may be involved in adipogenesis [40]; (iii) DIO2 is a candidate gene for CI3 (between 106921984 and 109413032 bp) because Dio2 knock-out mice were shown to be prone to obesity [41]; (iv) GALC was defined as a candidate gene for CI4 (between 110964904 and $116355960 \mathrm{bp}$ ) because it is part of a fine-mapped QTL ( 3 Mb) for intramuscular fat content [42]; (v) for CI5 (between 117489560 and 120450760 bp), we identified $T T C 7 B$ as a promising candidate gene because it was reported to be a candidate gene for obesity in mice [43]; (vi) for CI6 (between 121429704 and 124 338 264), the genes PRIMA1, FAM181A, ASB2, OTUB2, DDX24 and ISG12(A) overlapped with a chromosomal region that was reported to be associated with $10^{\text {th }}$ rib backfat by Fan et al. [39]; and (vii) for CI7 (between 125575968 and 130971280 bp), we identified $\mathrm{DIO} 3$ as a promising candidate gene since it was found to be associated with a number of fat deposition 


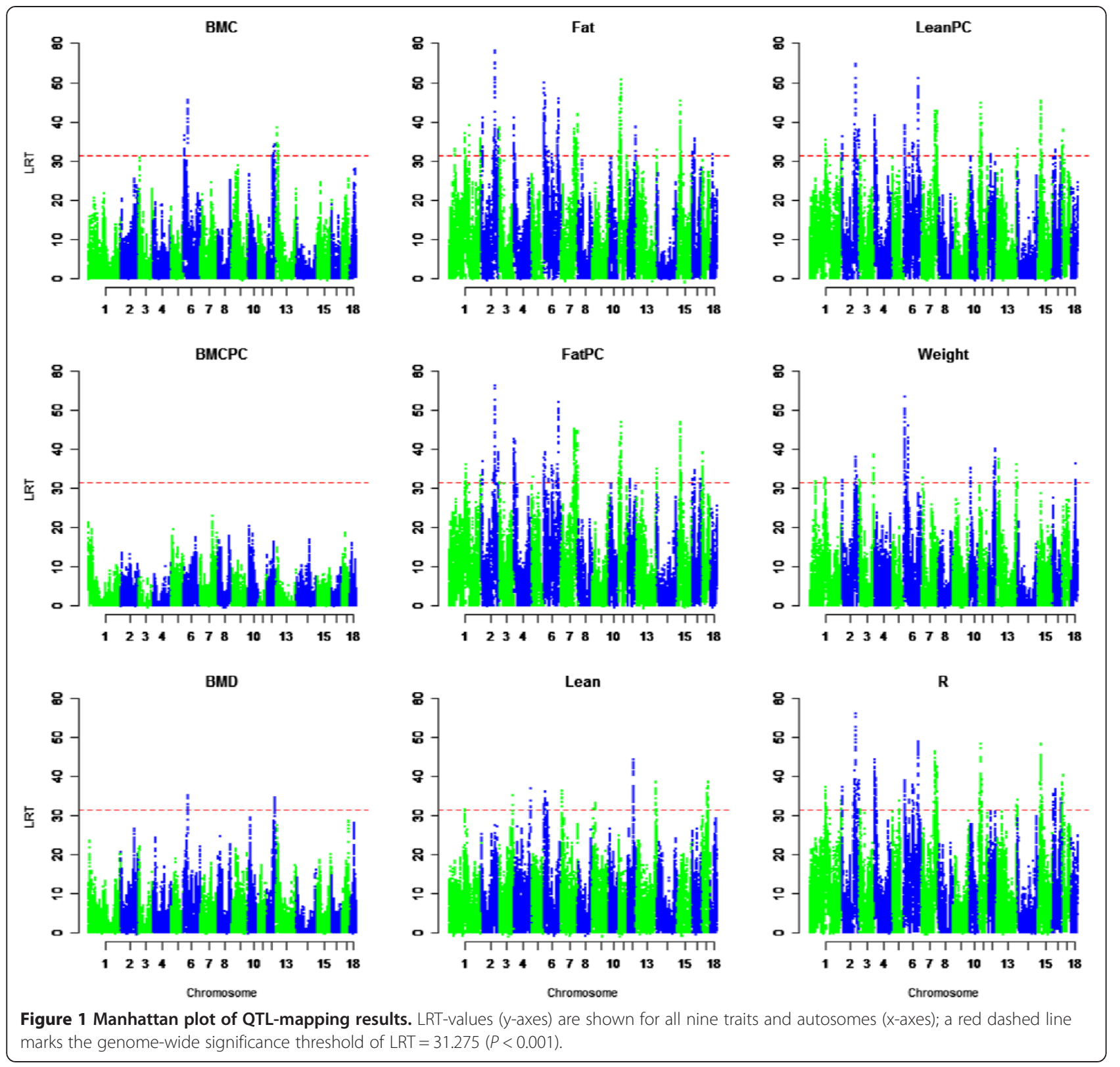

and carcass traits in pig [44]. However, without further analysis it is not possible to discriminate carryover effects from individual nearby QTL.

Candidate genes for the highest peaks across the genome All traits except BMCPC reached a genome-wide significance of $P<0.001$. The eight highest peaks across the genome (one for each significant trait) belonged to four QTL on three chromosomes (Table 2). The highest peaks of Fat (LRT = 58.1), FatPC (56.16), LeanPC (54.78) and R (55.82) were assigned to one of these QTL. Moreover, an additional significant peak for Weight (LRT = 37.899) fell within this confidence interval (Chr2:129 544 104-135 064 176).
Since the peak positions for Weight, at 133150512 bp, and for the other traits, at 133237232 bp, were only separated by two markers, it can be assumed that all significant associations identified in this region share a common mechanism. The gene that is closest to both peaks according to the Ensembl genome browser [45] is ZNF608, located between 133256788 and 133396887 bp. ZNF608 has been reported to be associated with body mass index (BMI) in humans [46,47]. Moreover, scribbler (sbb), which is the ZNF608 gene homologue in Drosophila [48], is suggested to be involved in larval food search behavior under starvation conditions [49]. Although the molecular mechanisms that underlie the function of ZNF608 are not 


\begin{tabular}{|c|c|c|c|c|c|c|c|c|c|}
\hline Chr & BMC & BMD & Fat & FatPC & Lean & LeanPC & Weight & $\mathbf{R}$ & Sum \\
\hline 1 & - & - & 5 & 5 & 1 & 3 & 2 & 5 & 21 \\
\hline 2 & - & - & 6 & 6 & - & 5 & 3 & 6 & 26 \\
\hline 3 & - & - & 1 & 1 & 2 & - & 4 & 1 & 9 \\
\hline 4 & - & - & 3 & 3 & 1 & 3 & - & 3 & 13 \\
\hline 5 & - & - & - & 1 & - & - & - & 1 & 2 \\
\hline 6 & 3 & 1 & 5 & 5 & 3 & 5 & 4 & 5 & 31 \\
\hline 7 & - & - & 5 & 6 & 1 & 4 & 1 & 6 & 23 \\
\hline 8 & - & - & - & - & - & - & - & - & 0 \\
\hline 9 & - & - & - & - & 2 & - & - & - & 2 \\
\hline 10 & - & - & - & - & - & - & 1 & - & 1 \\
\hline 11 & - & - & 5 & 4 & - & 3 & - & 3 & 15 \\
\hline 12 & 3 & 1 & 1 & 1 & 1 & 1 & 2 & - & 10 \\
\hline 13 & 2 & - & 1 & 1 & 1 & 1 & 2 & 1 & 9 \\
\hline 14 & - & - & - & - & - & - & - & - & 0 \\
\hline 15 & - & - & 3 & 3 & - & 4 & - & 3 & 13 \\
\hline 16 & - & - & 2 & 3 & - & 1 & - & 3 & 9 \\
\hline 17 & - & - & - & 2 & 3 & 2 & - & 2 & 9 \\
\hline 18 & - & - & 1 & - & - & - & 1 & - & 2 \\
\hline Total & 8 & 2 & 38 & 41 & 15 & 32 & 20 & 39 & 195 \\
\hline
\end{tabular}

clear [48], it seems to play a fundamental role in related traits across species. Therefore, ZNF608 can be regarded as a candidate gene associated with body composition in pigs. Since the five traits that have significant associations in this region are not independent and Fat has the highest LRT while the non-significant trait Lean had the lowest LRT among all traits for this region except BMCPC, it can be hypothesized that ZNF608 influences directly absolute fat mass and thereby indirectly the remaining traits (FatPC, LeanPC, R and Weight).

The highest peak for Weight $(\mathrm{LRT}=53.279)$ was observed at the beginning of chromosome 6 at $8723052 \mathrm{bp}$. The CI for this association (between 7334450 and $9257070 \mathrm{bp}$ ) included significant peaks for six other traits:
Lean $(\mathrm{LRT}=36.0)$, BMC (36.6), R (38.8), LeanPC (38.92), FatPC (39.1) and Fat (49.84). The genes closest to the peak based on NCBI Map Viewer are CDYL2 between 7681404 and $7695761 \mathrm{bp}$ at the proximal end and, in addition to a $5 S$ ribosomal RNA gene (between 9222444 and $9222549 \mathrm{bp})$, two genes near the distal end: CLEC3A between 9640139 and 9647945 bp and WWOX between 9684708 and 9744573 bp. Differential expression between human osteoarthritic and normal cartilage has been reported for CLEC3A [50] and WWOX [51], which indicates an association with bone formation. Thus, CLEC3A and $W W O X$ are promising candidate genes for BMC. Moreover, WWOX-deficient (-/-) mice die three weeks post-partum and display multiple postnatal defects, such as growth retardation and abnormalities in bone metabolism [52]. Therefore, it can be hypothesized that $W W O X$ variants could also affect growth in pigs and, thus, traits such as Weight, Lean, LeanPC, FatPC, Fat and the fat-related R.

Another QTL on chromosome 6 had its highest peak across the genome at 37403520 bp for BMC (45.56) and BMD (35.14). At exactly the same position, Weight had a significant peak with an even higher LRT-value (45.76) than BMC and BMD. The CI for this QTL, from 36937640 to $37714128 \mathrm{bp}$, has no annotated gene, neither in Ensembl nor in the NCBI Map Viewer [53]. However, according to Ensembl, the two nearest genes are less than 150 and $30 \mathrm{~kb}$ apart from the CI position, i.e. TSHZ3 between 36787929 and 36791170 bp and ZNF507 between 37742417 and 37767697 bp. No known information about ZNF507 function suggests that it could be a candidate gene for BMC or BMD, but TSHZ3 has been shown to be associated with both muscle differentiation [54] and lipid traits [55]. Thus, one hypothesis for this QTL could be that, assuming that both Fat and Lean are affected only moderately by a TSHZ3 variant in the same direction, there is no significant effect on either trait but the accumulated effects are significant for Weight. Since weight is known to influence bone mineral traits [34], a significant change in Weight might also have significant effects on BMD and BMC. For example, Scholz et al. [34] found a positive

Table 2 Highest peak identified across the genome for each trait

\begin{tabular}{llllllll}
\hline Trait & Chr & Peak & LRT & Cl Start & Cl End & Mb & Candidate genes \\
\hline Fat & 2 & 133237232 & 58.1 & 129544104 & 135064176 & 5.52 & ZNF608 \\
FatPC & 2 & 133237232 & 56.16 & 129544104 & 135064176 & 5.52 & ZNF608 \\
LeanPC & 2 & 133237232 & 54.78 & 129544104 & 135064176 & 5.52 & ZNF608 \\
R & 2 & 133237232 & 55.82 & 129544104 & 135064176 & 5.52 & ZNF608 \\
Weight & 6 & 8723052 & 53.279 & 7334450 & 9257070 & 1.92 & (WWOX) \\
BMC & 6 & 37403520 & 45.56 & 36937640 & 37714128 & 0.78 & - (TSHZ3) \\
BMD & 6 & 37403520 & 35.14 & 36937640 & 37714128 & 0.78 & $-($ TSHZ3) \\
Lean & 12 & 42949544 & 44.279 & 41217576 & 45554716 & 4.34 & chemokines, NF1 \\
\hline
\end{tabular}

Genes in parenthesis lie outside of the respective confidence interval $(\mathrm{Cl})$. 
correlation of 0.85 between soft tissue mass and BMD (in vivo: adjusted $\mathrm{R}^{2}=0.72$ ).

The highest peak for Lean (44.279) was identified on chromosome 12 at 42949544 bp. Within the CI for this association (between 41217576 and 45554716 bp), we also detected significant peaks for BMC (31.28 at $42637020 \mathrm{bp}$ ) and Weight (35.439 at $42365148 \mathrm{bp}$ ). The three peaks are spread over a region that contains 33 markers and includes the genes CCL1 and CCL11, CCL2 and CCL8, the novel protein coding gene ENSSSCG00000030066 and one pseudogene (ENSSSCG00000017724). For a number of chemokines, among which are CCL2, CCL8 and CCL11, increased expression has been demonstrated in adipose tissue of obese human patients. Thus, it was hypothesized that chemokines might be involved in promoting adipose tissue inflammation in obesity [56]. Moreover, a relationship between higher serum levels of the inflammatory chemokines CCL11 and CCL2 with lower lean body mass has been shown in geriatric patients [57]. NF1 is another interesting candidate gene within the CI of the peak for Lean. This gene is involved in neurofibromatosis type 1 in humans. Among other complications, affected individuals are likely to have low BMD, which is probably caused by NF1 haploinsufficiency [58,59]. Moreover, NF1 seems to be essential for muscle development and metabolism, because NF1 muscle-specific knock-out mice weigh significantly less than control mice [58]. Although NF1 lies within the CI of a peak for Lean only, it is a remarkable candidate gene that could be directly involved in not only Lean but also Weight and BMC.

\section{Additional candidate genes}

Besides the candidate genes mentioned in the previous section, we were able to define at least one candidate gene for most (49 out of 72) of the QTL. For reasons of clarity, we will concentrate only on some of these candidate genes in the following, but in Additional files 3, 4, $5,6,7,8,9$ and 10, all candidate genes are marked in bold letters.

\section{QTL associated with Fat, FatPC, LeanPC and $R$ traits}

The QTL region on chromosome 6 for Fat, FatPC, LeanPC and $\mathrm{R}$ spans more than $5 \mathrm{Mb}$ (between 133183304 and $138202064 \mathrm{bp})$. To date, this region contains more than 40 annotated genes based on Ensembl and NCBI Map Viewer. Among these, eight genes were considered as candidate genes for the associated traits: DIRAS3, PDE4B, LEPROT, (LEPR), DNAJC6, AK3L1, JAK1 and PGM1. For $D I R A S 3$, a correlation of the expression level with intramuscular fat has been confirmed in cattle [60]. The subregion that contains PDE4B, LEPROT, DNAJC6, AK3L1 and $J A K 1$ has been associated with backfat thickness in the Duroc pig breed [61] and a segment that includes LEPROT, DNAJC6, and AK3L1 was declared to be associated with intramuscular fat content in pigs [62]. PDE4B has been associated with backfat thickness in pigs and with obesity in humans $[7,62,63]$. In addition, Lee et al. [64] detected significant associations with backfat thickness for both $P D E 4 B$ and LEPROT. Although to date LEPR has not been positioned on the Sscrofa 10.2 assembly, it is assumed to be located next to LEPROT based on the human genome map and thus within the QTL region. For LEPR, significant associations with average daily weight gain and backfat thickness in Duroc pigs have been demonstrated [65]. Further evidence for a relation between $L E P R$ and fat-related traits is available from the literature, i.e. a highly significant differential expression of $L E P R$ in muscle tissue of pig breeds that display divergent obesity traits was reported by [66] and an association between a non-coding variation in LEPR and higher BMI was demonstrated in Native Americans [67]. Although the localization of LEPR within our QTL region needs to be confirmed, it represents a promising candidate gene, especially for fat-related traits. Another candidate gene in this QTL region, PGM1, has been shown to be significantly associated with BMI in humans [68]. Moreover, higher expression of PGM1 was detected in fast-growing compared to slow-growing chickens [69]. Overall, this QTL spans a chromosomal region that has been shown in many studies to be involved with, in particular, fat-related traits in pigs, cattle, humans, and mice. However, without further investigations we cannot narrow down the number of candidate genes or assess a possible interaction between several of these candidate genes. Thus, deciphering the cause of a QTL might be much easier for QTL that contain only few candidate genes. Some examples of such QTL will be discussed in the following.

\section{QTL associated with Weight}

Within a $2 \mathrm{Mb}$ QTL for Weight on chromosome 1, between 107871792 and 109893944 bp, which contains 20 genes, we identified two possible candidate genes: LIPG and $A C A A 2$. In humans, LIPG expression was shown to be increased in obese individuals compared to normal weight individuals [70]. Therefore, we hypothesize that LIPG may also be involved in lipid metabolism pathways in the pig and affect Weight. $A C A A 2$, the second candidate gene, encodes an enzyme that catalyzes the last step of the mitochondrial fatty acid beta-oxidation spiral [71]. Thus, since $A C A A 2$ is also involved in the pathway of fat metabolism, it may affect Weight.

\section{QTL associated with FAT}

Another 1.5 Mb QTL that spans almost 20 annotated genes was detected for Fat on chromosome 1 (between 38634124 and $40136908 \mathrm{bp}$ ). A possible candidate gene for this QTL is RSPO3 since loci near this gene were reported to be associated with waist-hip ratio in humans with European [72] or African ancestry [73]. 


\section{QTL associated with FatPC and $R$}

We mapped a 1.5 Mb QTL for FatPC and R (between 104 522416 and $105871296 \mathrm{bp}$ ) on chromosome 7. TGFB3 is the most promising candidate among the 13 genes in this chromosomal region based on results of Li et al. [74] who demonstrated an association between a polymorphism in the TGFB3 gene and growth and body composition traits in chickens, including weight of breast muscle and abdominal fat pad. Thus, it can be hypothesized that TGFB3 variants influence both fat and lean mass, which may cause a significant change in FatPC and consequently in $\mathrm{R}$.

\section{QTL associated with FatPC and LeanPC}

A QTL for FatPC and LeanPC that extended over less than $500 \mathrm{~kb}$ was detected on chromosome 12 (between 14714488 and $15183214 \mathrm{bp}$ ). We identified GH1 as an obvious candidate gene for this QTL since Fontanesi et al. [75] reported a significant association of a marker within the 3'-UTR region of GH1 with backfat thickness in the Italian Large White pig breed. In addition, a premature stop codon in GH1 causes an increased adipose tissue accumulation in zebrafish among other abnormalities (such as decreased somatic growth), which supports the importance of GH1 for fat-related traits, even across species [76].

\section{Comparison of our results with those of Fan et al.}

Finally, we develop some reflections on our results in the light of the findings of Fan et al. [39] since we detected numerous chromosomal regions that they had identified in their GWA study on body composition in pigs. Five of the chromosomal regions that Fan et al. [39] found to be associated with last rib backfat or $10^{\text {th }}$ rib backfat match QTL that we mapped for Fat or FatPC. Two other regions reported by Fan et al. [39] overlap with QTL for Lean and another one with a QTL for Weight. It should also be noted that three candidate chromosomal regions detected for body length, hip structure, and weak top line overlap with QTL for Weight in our study. All genes that overlap with regions reported by Fan et al. [39] are marked by asterisks in Additional files 3, 4, 5, 6, 7, 8, 9 and 10. A region that was associated with last rib backfat on chromosome 2 in Fan et al. [39] overlapped with one of our QTL for Fat, FatPC, LeanPC, Weight and, R (between 7012572 and 9 118948 bp). For this region, Fan et al. [39] did not suggest a candidate gene. Interestingly, annotation of our QTL with the currently known genes revealed two genes, RCOR2 (between 7350357 and 7352786 bp) and MARK2 (between 7355161 and 7364707 bp), within this region that were not reported by Fan et al. [39], which suggests that these genes were annotated in the pig genome only recently. Hurov et al. [77] showed that a loss of the MARK2 polarity kinase leads to decreased adiposity. No association with appropriate traits has been established for RCOR2. Thus, MARK2 is a candidate gene for both the
QTL detected in our study and the region associated with last rib backfat thickness in Fan et al. [39].

\section{Conclusions}

Our LDLA-based QTL mapping approach revealed significant peaks for all traits except for BMCPC. Most of the 72 identified QTL were associated with more than one trait and some of these associations confirmed previously reported results in pigs. However, we also detected significant associations in regions that have not been published before. Based on the literature, we identified candidate genes for most of the detected QTL and suggest a number of new and promising candidate genes, such as ZNF608 or WWOX.

\section{Additional files}

Additional file 1: 195 confidence intervals for 72 QTL. Description: For each of the 72 mapped QTL (consecutively numbered) the respective significant phenotype (PT), its peak position (Peak) in bp (chromosome: Chr) and the maximal LRT-value (LRT) are given. In addition, start (Cl start in bp) and end ( $\mathrm{Cl}$ end in bp) of each confidence interval and its size in $\mathrm{Mb}$ are given. QTL that are framed in red are those for which more than one PT was significant.

Additional file 2: Gene names. Description: This table provides the full names of all gene symbols mentioned within the main text.

Additional file 3: Genome-wide confidence intervals for BMC. Description: The consecutive QTL number based on Additional file 1 is given for each confidence interval (CI) as well as chromosome, peak-position with respective LRT-value, Cl position in bp and distance in Mb. Genes listed in the last column are all known, characterized genes according to Ensembl and NCBI genome browsers. Genes in bold characters are candidate genes that are based on a literature search for related traits and pathways in the pig and other species. Genes that were shown to be associated with related traits in Fan et al. [39] are marked with asterisks.

Additional file 4: Genome-wide confidence intervals for BMD. Description: The consecutive QTL number based on Additional file 1 is given for each confidence interval $(\mathrm{Cl})$ as well as chromosome, peakposition with respective LRT-value, Cl position in bp and distance in Mb. Genes listed in the last column are all known, characterized genes according to Ensembl and NCBI genome browsers. Genes in bold characters are candidate genes that are based on a literature search for related traits and pathways in the pig and other species. Genes that were shown to be associated with related traits in Fan et al. [39] are marked with asterisks.

Additional file 5: Genome-wide confidence intervals for Fat. Description: The consecutive QTL number based on Additional file 1 is given for each confidence interval (CI) as well as chromosome, peak-position with respective LRT-value, Cl position in bp and distance in Mb. Genes listed in the last column are all known, characterized genes according to Ensembl and NCBI genome browsers. Genes in bold characters are candidate genes that are based on a literature search for related traits and pathways in the pig and other species. Genes that were shown to be associated with related traits in Fan et al. [39] are marked with asterisks.

Additional file 6: Genome-wide confidence intervals for FatPC. Description: The consecutive QTL number based on Additional file 1 is given for each confidence interval (CI) as well as chromosome, peak-position with respective LRT-value, Cl position in bp and distance in Mb. Genes listed in the last column are all known, characterized genes according to Ensembl and NCBI genome browsers. Genes in bold characters are candidate genes that are based on a literature search for related traits and pathways in the pig and other species. Genes that were shown to be associated with related traits in Fan et al. [39] are marked with asterisks. 


\section{Additional file 7: Genome-wide confidence intervals for Lean.}

Description: The consecutive QTL number based on Additional file 1 is given for each confidence interval (Cl) as well as chromosome, peak-position with respective LRT-value, Cl position in bp and distance in Mb. Genes listed in the last column are all known, characterized genes according to Ensembl and NCBI genome browsers. Genes in bold characters are candidate genes that are based on a literature search for related traits and pathways in the pig and other species. Genes that were shown to be associated with related traits in Fan et al. [39] are marked with asterisks.

\section{Additional file 8: Genome-wide confidence intervals for LeanPC.}

Description: The consecutive QTL number based on Additional file 1 is given for each confidence interval (CI) as well as chromosome, peak-position with respective LRT-value, Cl position in bp and distance in Mb. Genes listed in the last column are all known, characterized genes according to Ensembl and NCBI genome browsers. Genes in bold characters are candidate genes that are based on a literature search for related traits and pathways in the pig and other species. Genes that were shown to be associated with related traits in Fan et al. [39] are marked with asterisks.

Additional file 9: Genome-wide confidence intervals for Weight. Description: The consecutive QTL number based on Additional file 1 is given for each confidence interval (Cl) as well as chromosome, peak-position with respective LRT-value, Cl position in bp and distance in Mb. Genes listed in the last column are all known, characterized genes according to Ensembl and NCBI genome browsers. Genes in bold characters are candidate genes that are based on a literature search for related traits and pathways in the pig and other species. Genes that were shown to be associated with related traits in Fan et al. [39] are marked with asterisks.

Additional file 10: Genome-wide confidence intervals for $\mathbf{R}$.

Description: The consecutive QTL number based on Additional file 1 is given for each confidence interval (Cl) as well as chromosome, peak-position with re spective LRT-value, $\mathrm{Cl}$ position in bp and distance in Mb. Genes listed in the last column are all known, characterized genes according to Ensembl and NCBl genome browsers. Genes in bold characters are candidate genes that are based on a literature search for related traits and pathways in the pig and other species. Genes that were shown to be associated with related traits in Fan et al. [39] are marked with asterisks.

\section{Competing interests}

The authors declare that they have no competing interests.

\section{Authors' contributions}

SR designed the mapping study, performed data analysis, interpreted data and drafted the manuscript. PK performed DXA analyses, blood sampling, veterinary control, and revised the manuscript. MB performed DXA analyses, blood sampling, veterinary control, and revised the manuscript. If contributed material and scientific advice on Cerdo Iberico and revised the manuscript. JP participated in data interpretation and helped to draft the manuscript. IM designed and coordinated the mapping study, contributed analysis tools, performed data analysis and critically revised the manuscript. AS designed and coordinated the study and critically revised the manuscript. All authors read and approved the final manuscript.

\section{Acknowledgments}

The authors thank colleagues for stimulating discussion, stockman staff for assistance with the DXA analysis, laboratory staff for technical assistance and reviewers and editor for helpful comments.

\section{Author details}

${ }^{1}$ Chair of Animal Genetics and Husbandry, Ludwig-Maximilians-University Munich, Veterinärstrasse 13, 80539 Munich, Germany. ${ }^{2}$ Department Agrikultur, University of Applied Sciences Weihenstephan-Triesdorf, Weidenbach, Germany. ${ }^{3}$ Livestock Center of the Faculty of Veterinary Medicine, Ludwig-Maximilians-University Munich, Oberschleissheim, Germany. ${ }^{4}$ Spanish National Research Council (CSIC), Granada, Spain.

Received: 10 April 2014 Accepted: 19 September 2014

Published online: 28 October 2014

\section{References}

1. Rothschild MF, Hu ZL, Jiang ZH: Advances in QTL mapping in pigs. Int J Biol Sci 2007, 3:192-197.

2. Rothschild MF, Ruvinsky A: The Genetics of the Pig. Oxon: CABI Press; 1998.

3. Ernst CW, Steibel JP: Molecular advances in QTL discovery and application in pig breeding. Trends Genet 2013, 29:215-224.

4. PigQTLdb. [http://www.animalgenome.org/cgi-bin/QTLdb/SS/index]

5. van Ooijen JW: Accuracy of mapping quantitative trait loci in autogamous species. Theor App/ Genet 1992, 84:803-811.

6. Do DN, Strathe AB, Ostersen T, Jensen J, Mark T, Kadarmideen HN Genome-wide association study reveals genetic architecture of eating behavior in pigs and its implications for humans obesity by comparative mapping. PLoS One 2013, 8:e71509.

7. Fontanesi L, Schiavo G, Galimberti G, Calo DG, Scotti E, Martelli PL, Buttazzoni L, Casadio R, Russo V: A genome wide association study for backfat thickness in Italian Large White pigs highlights new regions affecting fat deposition including neuronal genes. BMC Genomics 2012, 13:583.

8. Fowler KE, Pong-Wong R, Bauer J, Clemente EJ, Reitter CP, Affara NA, Waite S, Walling GA, Griffin DK: Genome wide analysis reveals single nucleotide polymorphisms associated with fatness and putative novel copy number variants in three pig breeds. BMC Genomics 2013, 14:784.

9. Grindflek $E_{\text {, Lien }}$, Hamland $H$, Hansen MHS, Kent M, van Son M, Meuwissen THE: Large scale genome-wide association and LDLA mapping study identifies QTLs for boar taint and related sex steroids. BMC Genomics 2011, 12:362.

10. Ma JW, Yang J, Zhou LS, Zhang ZY, Ma HB, Xie XH, Zhang F, Xiong XW, Cui LL, Yang H, Liu XX, Duan YY, Xiao SJ, Ai HS, Ren J, Huang LS: Genome-wide association study of meat quality traits in a white Duroc $x$ Erhualian F2 intercross and Chinese Sutai pigs. PLoS One 2013, 8:e64047.

11. Kogelman LJA, Kadarmideen HN, Mark T, Karlskov-Mortensen P, Bruun CS, Cirera S, Jacobsen MJ, Jørgensen CB, Fredholm M: An F2 pig resource population as a model for genetic studies of obesity and obesity-related diseases in humans: Design and genetic parameters. Front Genet 2013, 4:29.

12. Chan RSM, Woo J: Prevention of overweight and obesity: How effective is the current public health approach. Int J Environ Res Public Health 2010 7:765-783.

13. World Health Organization: Obesity and overweight (Fact sheet $\mathrm{N}^{\circ} 311$ ). [http://www.who.int/mediacentre/factsheets/fs311/en/]

14. Scholz AM, Förster M: Genauigkeit der Dualenergie-Röntgenabsorptiometrie (DXA) zur Ermittlung der Körperzusammensetzung von Schweinen in vivo. Arch Tierz 2006, 49:462-476.

15. Kremer PV, Fernandez-Figares I, Förster M, Scholz AM: In vivo body composition in autochthonous and conventional pig breeding groups by dual-energy $\mathrm{X}$-ray absorptiometry and magnetic resonance imaging under special consideration of Cerdo Iberico. Animal 2012, 6:2041-2047.

16. Laenoi W, Uddin MJ, Cinar MU, Grosse-Brinkhaus C, Tesfaye D, Jonas E, Scholz AM, Tholen E, Looft C, Wimmers K, Phatsara C, Juengst H, Sauerwein $\mathrm{H}$, Mielenz M, Schellander K: Quantitative trait loci analysis for leg weakness-related traits in a Duroc x Pietrain crossbred population. Genet Sel Evol 2011, 43:13.

17. Laenoi W, Uddin MJ, Cinar MU, Phatsara C, Tesfaye D, Scholz AM, Tholen E, Looft C, Mielenz M, Sauerwein H, Schellander K: Molecular characterization and methylation study of matrix gla protein in articular cartilage from pig with osteochondrosis. Gene 2010, 459:24-31.

18. Rangkasenee N, Murani E, Brunner R, Schellander K, Cinar MU, Scholz AM, Luther H, Hofer A, Ponsuksili S, Wimmers K: KRT8, FAF1 and PTH1R gene polymorphisms are associated with leg weakness traits in pigs. Mol Biol Rep 2013, 40:2859-2866.

19. Yazdi MH, Lundeheim N, Rydhmer L, Ringmar-Cederberg E, Johansson K Survival of Swedish Landrace and Yorkshire sows in relation to osteochondrosis: a genetic study. Anim Sci 2000, 71:1-9.

20. Fukawa K, Kusuhara S: The genetic and non-genetic aspects of leg weakness and osteochondrosis in pigs - Review. Asian Australas J Anim Sci 2001, 14:114-122.

21. Pig genome 10.2. [http://www.animalgenome.org/repository/pig/ Genome_10.2_mappings/]

22. Browning $B L$, Browning SR: A unified approach to genotype imputation and haplotype-phase inference for large data sets of trios and unrelated individuals. Am J Hum Genet 2009, 84:210-223. 
23. Powell JE, Visscher PM, Goddard ME: Reconciling the analysis of IBD and IBS in complex trait studies. Nat Rev Genet 2010, 11:800-805.

24. R Core Team: R: A Language and Environment for Statistical Computing. Vienna, Austria: R Foundation for Statistical Computing; 2013.

25. Dinno A: paran: Horn's Test of Principal Components/Factors. http://cran. r-project.org/web/packages/paran/index.html.

26. Meuwissen THE, Goddard ME: Multipoint identity-by-descent prediction using dense markers to map quantitative trait loci and estimate effective population size. Genetics 2007, 176:2551-2560.

27. Lee SH, Van der Werf JHJ: Using dominance relationship coefficients based on linkage disequilibrium and linkage with a general complex pedigree to increase mapping resolution. Genetics 2006, 174:1009-1016.

28. Meuwissen THE, Karlsen A, Lien S, Olsaker I, Goddard ME: Fine mapping of a quantitative trait locus for twinning rate using combined linkage and linkage disequilibrium mapping. Genetics 2002, 161:373-379.

29. Gilmour AR, Gogel BJ, Cullis BR, Thompson R: ASReml User Guide. (Release 3.0). [http://vsni.de/downloads/asreml/release3/UserGuide.pdf]

30. Olsen HG, Lien S, Svendsen M, Nilsen H, Roseth A, Opsal MA, Meuwissen THE: Fine mapping of milk production QTL on BTA6 by combined linkage and linkage disequilibrium analysis. J Dairy Sci 2004, 87:690-698.

31. Visscher PM, Goddard ME: Prediction of the confidence interval of quantitative trait loci location. Behav Genet 2004, 34:477-482.

32. Svendsen OL, Haarbo J, Hassager C, Christiansen C: Accuracy of measurements of body-composition by dual-energy X-ray absorptiometry in vivo. Am J Clin Nutr 1993, 57:605-608.

33. Mitchell AD, Scholz A, Pursel V: Prediction of the in vivo body composition of pigs based on cross-sectional region analysis of dual energy X-ray absorptiometry (DXA) scans. Arch Tierz 2002, 45:535-545.

34. Scholz AM, Mitchell AD, Förster M, Pursel VG: Two-site evaluation of the relationship between in vivo and carcass dual energy $\mathrm{X}$-ray absorptiometry (DXA) in pigs. Livest Sci 2007, 110:1-11.

35. Zhao $L$, Jiang H, Papasian CJ, Maulik D, Drees B, Hamilton J, Deng HW: Correlation of obesity and osteoporosis: effect of fat mass on the determination of osteoporosis. J Bone Miner Res 2008, 23:17-29.

36. Cao JJ: Effects of obesity on bone metabolism. J Orthop Surg Res 2011, 6:30.

37. Meuwissen THE, Goddard ME: Mapping multiple QTL using linkage disequilibrium and linkage analysis information and multitrait data. Genet Sel Evol 2004, 36:261-279.

38. Olsen HG, Lien $S$, Gautier M, Nilsen H, Roseth A, Berg PR, Sundsaasen KK, Svendsen M, Meuwissen THE: Mapping of a milk production quantitative trait locus to a 420-kb region on bovine chromosome 6. Genetics 2005, 169:275-283.

39. Fan B, Onteru SK, Du ZQ, Garrick DJ, Stalder KJ, Rothschild MF: Genomewide association study identifies loci for body composition and structural soundness traits in pigs. PLoS One 2011, 6:e14726.

40. Du BW, Cawthorn WP, Su A, Doucette CR, Yao Y, Hemati N, Kampert S, McCoin C, Broome DT, Rosen CJ, Yang GS, MacDougald OA: The transcription factor paired-related Homeobox 1 (Prrx1) inhibits adipogenesis by activating transforming growth factor-beta (TGF beta) signaling. J Biol Chem 2013, 288:3036-3047.

41. Marsili A, Aguayo-Mazzucato C, Chen T, Kumar A, Chung M, Lunsford EP, Harney JW, Thuy VT, Gianetti E, Ramadan W, Chou C, Bonner-Weir S, Larsen PR, Silva JE, Zavacki AM: Mice with a targeted deletion of the Type 2 Deiodinase are insulin resistant and susceptible to diet induced obesity. PLoS One 2011, 6:e20832.

42. Sato $S$, Hasebe H, Sato S, Asahi Y, Hayashi T, Kobayashi E, Sugimoto Y: High-resolution physical mapping and construction of a porcine contig spanning the intramuscular fat content QTL. Anim Genet 2006, 37:113-120.

43. Morton NM, Nelson YB, Michailidou Z, Di Rollo EM, Ramage L, Hadoke PWF, Seckl JR, Bunger L, Horvat S, Kenyon CJ, Dunbar DR: A stratified transcriptomics analysis of polygenic fat and lean mouse adipose tissues identifies novel candidate obesity genes. PLOS One 2011, 6:e23944.

44. Qiao M, Wu HY, Guo L, Mei SQ, Zhang PP, Li FE, Zheng R, Deng CY: Imprinting analysis of porcine $\mathrm{DIO} 3$ gene in two fetal stages and association analysis with carcass and meat quality traits. Mol Biol Rep 2012, 39:2329-2335.

45. Ensembl. [http://www.ensembl.org/Sus_scrofa/Info/Index]

46. Ntalla I, Panoutsopoulou K, Vlachou P, Southam L, William Rayner N, Zeggini E, Dedoussis GV: Replication of established common genetic variants for adult $\mathrm{BMI}$ and childhood obesity in Greek adolescents: The TEENAGE study. Ann Hum Genet 2013, 77:268-274.
47. Speliotes EK, Willer CJ, Berndt SI, Monda KL, Thorleifsson G, Jackson AU, Allen HL, Lindgren CM, Luan JA, Magi R, Randall JC, Vedantam S, Winkler TW, Qi L, Workalemahu T, Heid IM, Steinthorsdottir V, Stringham HM, Weedon MN, Wheeler E, Wood AR, Ferreira T, Weyant RJ, Segre AV, Estrada K, Liang L, Nemesh J, Park J-H, Gustafsson S, Kilpelainen TO, et al: Association analyses of 249,796 individuals reveal 18 new loci associated with body mass index. Nat Genet 2010, 42:937-948.

48. Williams MJ, Almen MS, Fredriksson R, Schiöth HB: What model organisms and interactomics can reveal about the genetics of human obesity. Cell Mol Life Sci 2012, 69:3819-3834

49. Yang P, Shaver SA, Hilliker AJ, Sokolowski MB: Abnormal turning behavior in Drosophila larvae. Identification and molecular analysis of scribbler (sbb). Genetics 2000, 155:1161-1174.

50. Karlsson C, Dehne T, Lindahl A, Brittberg M, Pruss A, Sittinger M, Ringe J: Genome-wide expression profiling reveals new candidate genes associated with osteoarthritis. Osteoarthritis Cartilage 2010, 18:581-592.

51. Niopoulos D, Malizos KN, Oikonomou P, Tsezou A: Integrative microRNA and proteomic approaches identify novel osteoarthritis genes and their collaborative metabolic and inflammatory networks. PLoS One 2008, 3:e3740.

52. Aqeilan RI, Hassan MQ, de Bruin A, Hagan JP, Volinia S, Palumbo T, Hussain S, Lee SH, Gaur T, Stein GS, Lian JB, Croce CM: The WWOX tumor suppressor is essential for postnatal survival and normal bone metabolism. J Biol Chem 2008, 283:21629-21639.

53. NCBI Map Viewer. [http://www.ncbi.nlm.nih.gov/projects/mapview/maps. cgi?taxid $=9823 \&$ chr $=1]$

54. Faralli H, Martin E, Core N, Liu QC, Filippi P, Dilworth FJ, Caubit X, Fasano L: Teashirt-3, a novel regulator of muscle differentiation, associates with BRG1-associated factor 57 (BAF57) to inhibit myogenin gene expression. J Biol Chem 2011, 286:23498-23510

55. Dong CH, Beecham A, Wang LY, Slifer S, Wright CB, Blanton SH, Rundek T, Sacco RL: Genetic loci for blood lipid levels identified by linkage and association analyses in Caribbean Hispanics. J Lipid Res 2011, 52:1411-1419.

56. Huber J, Kiefer FW, Zeyda M, Ludvik B, Silberhumer GR, Prager G, Zlabinger GJ, Stulnig TM: CC chemokine and CC chemokine receptor profiles in visceral and subcutaneous adipose tissue are altered in human obesity. $J$ Clin Endocrinol Metab 2008, 93:3215-3221.

57. Beyer I, Njemini R, Bautmans I, Demanet C, Bergmann P, Mets T: Inflammation-related muscle weakness and fatigue in geriatric patients. Exp Gerontol 2012, 47:52-59.

58. Sullivan K, El-Hoss J, Quinlan KG, Deo N, Garton F, Seto JT, Gdalevitch M, Turner N, Cooney GJ, Kolanczyk M, North KN, Little DG, Schindeler A: NF1 is a critical regulator of muscle development and metabolism. Hum $\mathrm{Mol}$ Genet 2014, 23:1250-1259.

59. Yu X, Chen S, Potter OL, Murthy SM, Li J, Pulcini JM, Ohashi N, Winata T, Everett ET, Ingram D, Clapp WD, Hock JM: Neurofibromin and its inactivation of Ras are prerequisites for osteoblast functioning. Bone 2005, 36:793-802.

60. Sadkowski T, Ciecierska A, Majewska A, Oprzadek J, Dasiewicz K, Ollik M, Wicik Z, Motyl T: Transcriptional background of beef marbling - Novel genes implicated in intramuscular fat deposition. Meat Sci 2014, 97:32-41.

61. Okumura N, Matsumoto T, Hayashi T, Hirose K, Fukawa K, Itou T, Uenishi H, Mikawa S, Awata T: Genomic regions affecting backfat thickness and cannon bone circumference identified by genome-wide association study in a Duroc pig population. Anim Genet 2013, 44:454-457.

62. Kim JH, Ovilo C, Park EW, Fernandez A, Lee JH, Jeon JT, Lee JG: Minimizing a QTL region for intramuscular fat content by characterizing the porcine Phosphodiesterase 4B (PDE4B) gene. BMB Rep 2008, 41:466-471.

63. Lee KT, Byun MJ, Kang KS, Park EW, Lee SH, Cho S, Kim H, Kim KW, Lee T, Park JE, Park W, Shin D, Park HS, Jeon JT, Choi BH, Jang GW, Choi SH, Kim DW, Lim D, Park HS, Park MR, Ott J, Schook LB, Kim TH, Kim H: Neuronal genes for subcutaneous fat thickness in human and pig are identified by local genomic sequencing and combined SNP association study. PLOS One 2011, 6:e16356.

64. Lee KT, Byun MJ, Kang KS, Hwang H, Park EW, Kim JM, Kim TH, Lee SH: Single nucleotide polymorphism association study for backfat and intramuscular fat content in the region between SW2098 and SW1881 on pig chromosome 6. J Anim Sci 2012, 90:1081-1087.

65. Hirose K, Ito T, Fukawa K, Arakawa A, Mikawa S, Hayashi Y, Tanaka K: Evaluation of effects of multiple candidate genes (LEP, LEPR, MC4R, PIK3C3, and VRTN) on production traits in Duroc pigs. Anim Sci J 2014, 85:198-206. 
66. Cirera S, Jensen MS, Elbrond VS, Moesgaard SG, Christoffersen BO,

Kadarmideen HN, Skovgaard K, Bruun CV, Karlskov-Mortensen P, Jorgensen CB, Fredholm M: Expression studies of six human obesity-related genes in seven tissues from divergent pig breeds. Anim Genet 2013, 45:59-66.

67. Traurig MT, Perez JM, Ma L, Bian L, Kobes S, Hanson RL, Knowler WC, Krakoff $J A$, Bogardus C, Baier LJ: Variants in the LEPR gene are nominally associated with higher BMI and lower 24-h energy expenditure in Pima Indians. Obesity 2012, 20:2426-2430

68. Gloria-Bottini F, Magrini A, Antonacci E, La Torre M, Di Renzo L, De Lorenzo A, Bergamaschi A, Bottini E: Phosphoglucomutase genetic polymorphism and body mass. Am J Med Sci 2007, 334:421-425.

69. Claire D'Andre H, Paul W, Shen X, Jia X, Zhang R, Sun L, Zhang X: Identification and characterization of genes that control fat deposition in chickens. J Anim Sci Biotechnol 2013, 4:43.

70. Miksztowicz V, Schreier L, McCoy M, Lucero D, Fassio E, Billheimer J, Rader DJ, Berg G: Role of SN1 lipases on plasma lipids in metabolic syndrome and obesity. Arterioscler Thromb Vasc Biol 2014, 34:669-675.

71. Dunning KR, Anastasi MR, Zhang VJ, Russell DL, Robker RL: Regulation of fatty acid oxidation in mouse cumulus-oocyte complexes during maturation and modulation by PPAR agonists. PLoS One 2014, 9:e87327.

72. Heid IM, Jackson AU, Randall JC, Winkler TW, Qi L, Steinthorsdottir V, Thorleifsson G, Zillikens MC, Speliotes EK, Magi R, Workalemahu T, White CC, Bouatia-Naji N, Harris TB, Berndt SI, Ingelsson E, Willer CJ, Weedon MN, Luan J, Vedantam S, Esko T, Kilpelainen TO, Kutalik Z, Li S, Monda KL, Dixon AL, Holmes CC, Kaplan LM, Liang L, Min JL, et al: Meta-analysis identifies 13 new loci associated with waist-hip ratio and reveals sexual dimorphism in the genetic basis of fat distribution. Nat Genet 2010, 42:949-960.

73. Liu CT, Monda KL, Taylor KC, Lange L, Demerath EW, Palmas W, Wojczynski MK, Ellis JC, Vitolins MZ, Liu S, Papanicolaou GJ, Irvin MR, Xue L, Griffin PJ, Nalls MA, Adeyemo A, Liu J, Li G, Ruiz-Narvaez EA, Chen WM, Chen F, Henderson BE, Millikan RC, Ambrosone CB, Strom SS, Guo X, Andrews JS, Sun W, Mosley TH, Yanek LR, et al: Genome-wide association of body fat distribution in African ancestry populations suggests new loci. PLoS Genet 2013, 9:e1003681.

74. Li H, Deeb N, Zhou H, Mitchell AD, Ashwell CM, Lamont SJ: Chicken quantitative trait loci for growth and body composition associated with transforming growth factor-beta genes. Poult Sci 2003, 82:347-356.

75. Fontanesi L, Galimberti G, Calo DG, Fronza R, Martelli PL, Scotti E, Colombo M, Schiavo G, Casadio R, Buttazzoni L, Russo V: Identification and association analysis of several hundred single nucleotide polymorphisms within candidate genes for back fat thickness in Italian Large White pigs using a selective genotyping approach. J Anim Sci 2012, 90:2450-2464.

76. McMenamin SK, Minchin JEN, Gordon TN, Rawls JF, Parichy DM: Dwarfism and increased adiposity in the gh1 mutant zebrafish vizzini. Endocrinology 2013, 154:1476-1487.

77. Hurov JB, Huang M, White LS, Lennerz J, Choi CS, Cho YR, Kim HJ, Priori JL, Piwnica-Worms D, Cantley LC, Kim JK, Shulman Gl, Piwnica-Worms H: Loss of the Par-1b/MARK2 polarity kinase leads to increased metabolic rate, decreased adiposity, and insulin hypersensitivity in vivo. Proc Natl Acad Sci U S A 2007, 104:5680-5685.

doi:10.1186/s12711-014-0068-2

Cite this article as: Rothammer et al:: Genome-wide QTL mapping of nine body composition and bone mineral density traits in pigs. Genetics Selection Evolution 2014 46:68.

\section{Submit your next manuscript to BioMed Central and take full advantage of:}

- Convenient online submission

- Thorough peer review

- No space constraints or color figure charges

- Immediate publication on acceptance

- Inclusion in PubMed, CAS, Scopus and Google Scholar

- Research which is freely available for redistribution

Submit your manuscript at www.biomedcentral.com/submit 